\title{
Vertical vs. Horizontal Packaging Design: Investigating the Effects of Packaging Form on Consumers' Perception of Utilitarian Food Products: An Abstract
}

\author{
Klaus-Peter Wiedmann, Jannick Bettels, and Janina Haase
}

\begin{abstract}
Packaging is one of the most powerful mediums for firms to appeal to the consumer because of its presence in the crucial moment of buying decision. Especially packaging form can be an effective instrument to stand out in the market. In particular in the food industry, representing a highly competitive market, packaging design represents an essential issue. Most firms use a packaging form that is squared and prototypical within their product category. However, the question arises if changing the packaging alignment may give a competitive edge. The objective of this paper is to examine the differences between vertical and horizontal packaging with regard to consumer perception and behavior. For this purpose, we present an experiment in the context of utilitarian food products. The results show that the vertical package elicits higher perceived aesthetics based on prototypicality in the given product category and more estimated package content due to the elongation bias. In contrast, the horizontal package evokes a higher perceived utilitarian value in consequence of an enhanced efficiency when processing the packaging text (utilitarian claims) horizontally. This effect surpasses the first two effects, so that finally the use of a horizontal package leads to a higher willingness to pay.
\end{abstract}

K.-P. Wiedmann $\cdot$ J. Bettels $(\bowtie) \cdot J$. Haase

Leibniz University of Hannover, Hannover, Germany

e-mail: wiedmann@m2.uni-hannover.de; bettels@m2.uni-hannover.de;

haase@m2.uni-hannover.de 